\title{
URETROPLASTIA DORSAL CON INJERTO LIBRE DE MUCOSA PREPUCIAL (TECNICA DE BARBAGLI): RESULTADOS PRELIMINARES DE NUESTRA SERIE.
}

\author{
Francisco Javier Vicente Prados, Miguel Tallada Buñuel, José Luis Rosales Leal, Beatriz Honrubia \\ Vílchez, Antonio Fernández Sánchez, Fernando Vázquez Alonso, Manrique Pascual Geler \\ Antonio Martínez Morcillo, Francisco Rodríguez Herrera, Eduardo Espejo Maldonado y \\ José Manuel Cozar Olmo.
}

Servicio de Urología del Hospital Universitario Virgen de las Nieves. Granada. España.

\begin{abstract}
Resumen.- OBJETIVO: Valoramos la indicación y nuestros resultados en el tratamiento de la estenosis de uretra compleja mediante la Técnica de Barbagli.

MÉTODO: Realizamos una revisión retrospectiva de 8 pacientes a los que se indica la uretroplastia dorsal con injerto de mucosa libre prepucial. Analizamos las características preoperatorias de los pacientes. La longitud media de la estenosis fue de $6,25 \mathrm{~cm}$, la localización bulbar en 7 casos y peneana en uno. El flujo medio obtenido en la flujometría preoperatoria fue de 8,78 $\mathrm{ml} / \mathrm{seg}$. Describimos la técnica y presentamos los resultados obtenidos con la misma.
\end{abstract}

RESULTADOS: Con un tiempo medio de seguimiento de 22 meses tenemos un 87,5\% de buenos resultados, entendiendo estos como la resolución definitiva de la estenosis. Sólo 1 paciente ha precisado un nuevo tratamiento quirúrgico (anastomosis término terminal), con buen resultado evolutivo.

CONCLUSIONES: Concluimos que la uretroplastia dorsal con injerto libre de mucosa prepucial es una técnica efectiva en estenosis de uretra larga y compleja y que por los resultados obtenidos, la consideramos técnica de primera elección en este tipo de estenosis uretrales.

Palabras clave: Estenosis uretral. Uretroplastia dorsal. Mucosa prepucial.

Summary.- OBJECTIVES: We evaluate the indication and results in the treatment of complex urethral stenosis by the Barbagli's technique.

METHODS: We retrospectively review 8 patients undergoing dorsal onlay urethroplasty with foreskin mucosa free graft. We analyze their preoperative characteristics. Mean stenosis length was $6.25 \mathrm{~cm}$ and the site was bulbar urethra in seven cases and penile in one. Mean preoperative flow was $8.78 \mathrm{ml} / \mathrm{sec}$. We describe the technique and present our results.

RESULTS: There are $87.5 \%$ of goods results (cure of the stenosis) with 22 months of mean follow-up. Only one patient required a second operation (end to end anastomosis) with good outcome.

CONCLUSIONS: The dorsal onlay urethroplasty with foreskin mucosa free graft is an effective technique in 
long and complex urethral stenosis, and we consider it the technique of first choice in view of our results.

Keywords: Urethral stenosis. Dorsal urethroplasty. Foreskin mucosa.

\section{INTRODUCCIÓN}

Desde que Barbagli describiera la técnica de uretroplastia dorsal con injerto libre de mucosa en 1995-1996, se confirman tasas de éxito del 85 al $97 \%$, por lo que nosotros la incluimos dentro de nuestro armamentario quirúrgico desde el año 2000.

Valoramos nuestra serie, evaluando las características preoperatorias de los 8 casos tratados, la

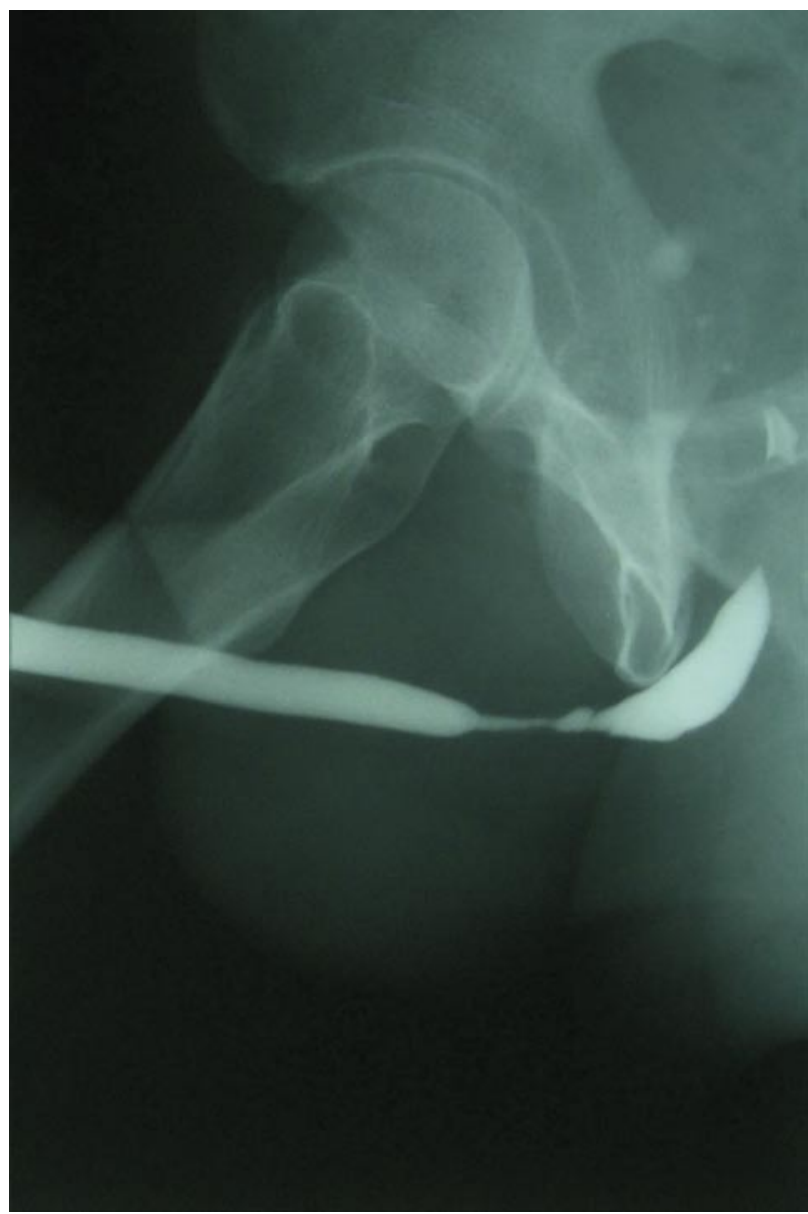

FIGURA 1. Uretrografía retrógrada: estenosis de uretra bulbar larga y arrosariada. técnica utilizada, las complicaciones, los resultados y los fracasos; así como el manejo de los mismos.

\section{MATERIAL Y MÉTODOS}

Hemos realizado una revisión retrospectiva de 8 pacientes que presentaban una estenosis uretral larga 7 a nivel bulbar y uno a nivel de uretra peneana) y tratados entre el año 2000 y el 2005 con uretroplastia dorsal e injerto libre de mucosa prepucial.

El estudio preoperatorio comprendió anamnesis, exploración física, cistouretrografía miccional y retrógrada y flujometría miccional aislada.

El estudio radiológico se realizó en todos los casos y la longitud media de la estenosis fue de 6,25 $\mathrm{cm}(3-8)$ en los casos de localización bulbar (Figura 1) y de $3 \mathrm{~cm}$ en el único caso de localización peneana.

La valoración funcional previa a la intervención mediante uroflujometría demostró un flujo máximo medio de $8,78 \mathrm{ml} / \mathrm{sg}(5-9,8)$ con volumen miccional medio de $216,62 \mathrm{ml}$, y un tiempo de flujo prolongado, con un patrón en meseta característico. (Figura 2).

En todos los pacientes se investigó urocultivo con antibiograma preoperatorio. En los que éste fue negativo se pautó profilaxis antibiótica con 1500 mgrs de cefuroxima acetilo 2 horas antes de la intervención. Cuando el cultivo fue positivo se trató la infección según el antibiograma.

En cuatro casos se indicó la uretroplastia de revestimiento dorsal como técnica quirúrgica de primera elección. En los cuatro restantes los pacientes presentaban una historia de uretrotomía endoscópica y/o dilataciones uretrales periódicas tras el fracaso de la técnica endoscópica.

El acceso quirúrgico fue perineal en los 7 casos de localización bulbar. Se valoró, con anterioridad, la existencia de mucosa prepucial suficiente, sobre todo ante antecedentes de postectomía (2 casos). Un paciente presentaba además una incurvación congénita ventral mayor de 60 grados que se resolvió con posterioridad a la cirugía uretral.

En quirófano se realiza lavado, con betadine jabonoso, abundante en la región perineal (previamente rasurada) y en la zona del glande-prepucio de donde se obtendrá el injerto.

Se lleva a cabo una liberación amplia de la uretra hasta porción sana proximal y distal $(1 \mathrm{~cm})$ a 


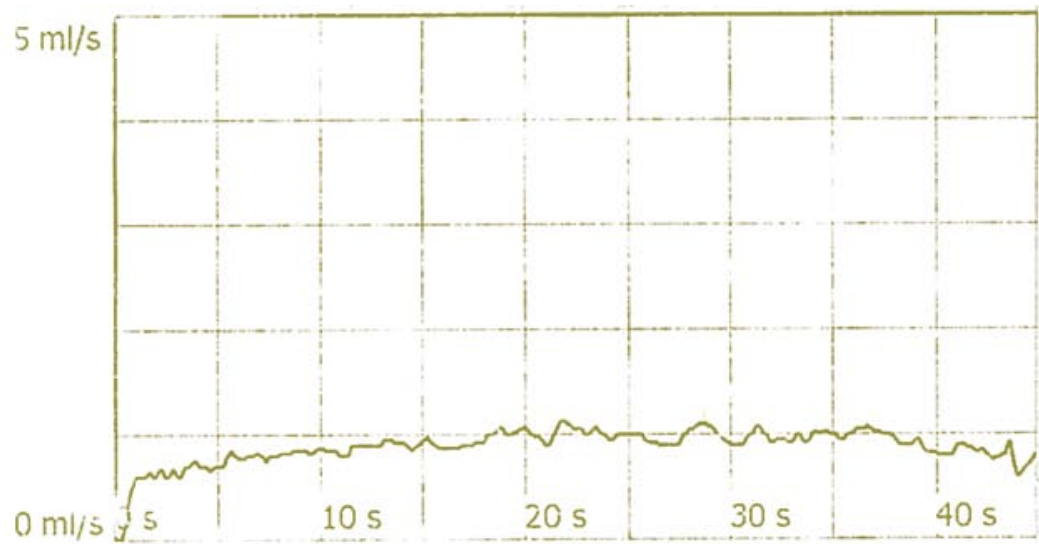

\section{Resultados}

12-11-2004 12:14

Tiempo de evacua.: $\quad 77 \mathrm{~s}$

Tiempo de flujo: $\quad 74 \mathrm{~s}$

Tiempo hasta el flujo máx: $59 \mathrm{~s}$

Flujo máximo:

$6.6 \mathrm{ml} / \mathrm{s}$

Flujo medio:

$3.9 \mathrm{ml} / \mathrm{s}$

Volumen vaciado:

$295 \mathrm{ml}$

Filtro:

Sensor:

Estándar

Disco giratorio

FIGURA 2. Uroflujometría: Qmáx de 6,6 ml/seg con un volumen de $295 \mathrm{ml}$. Típica morfología en meseta.

la estenosis. Una vez liberada se rota $180^{\circ}$ exponiendo su cara dorsal. (Figuras $3 a$ y $3 b$ ).

Abrimos longitudinalmente la zona estenótica hasta llegar a uretra sana. Comprobamos el calibre de la uretra proximal y distal midiendo la longitud exacta de la estenosis. (Figuras $4 a$ y $4 b$ ).

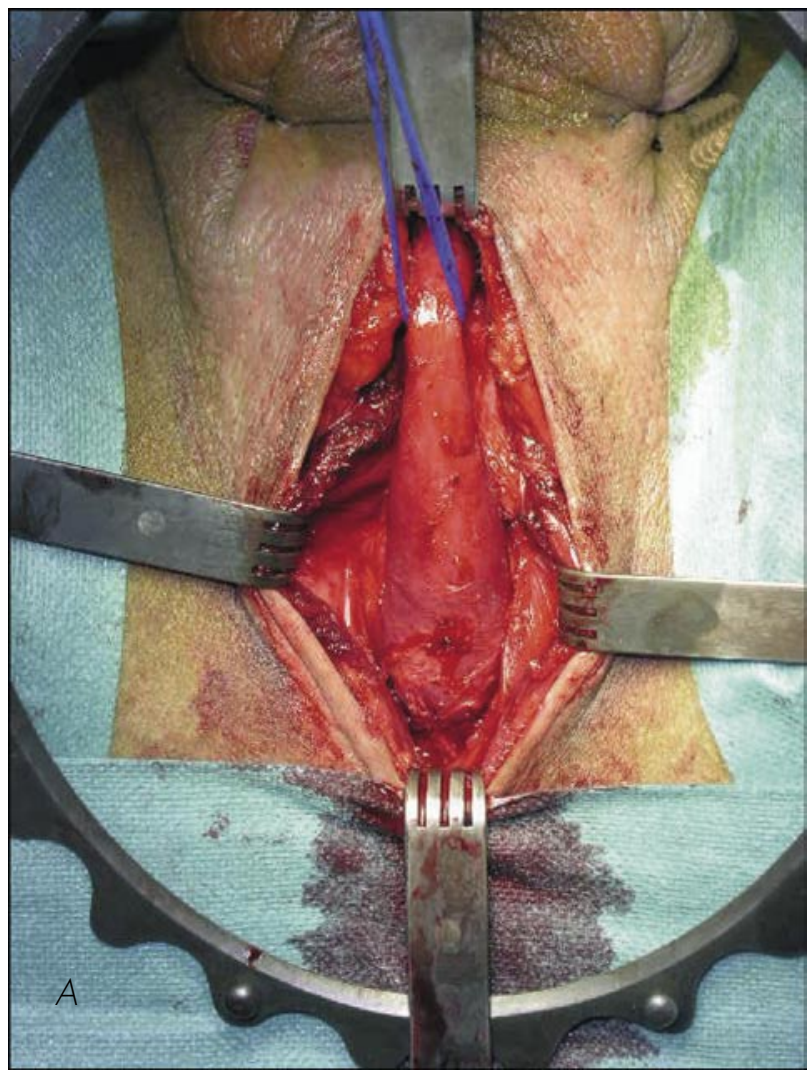

Obtenemos el injerto libre de mucosa prepucial de $2 \mathrm{cms}$ de ancho con una longitud igual o mayor a la de la estenosis, quedando disponible tras su preparación en banco. (Figuras $5 a$ y $5 b$ ).

El injerto se coloca a nivel de la albugínea de los cuerpos cavernosos y se estabiliza con puntos

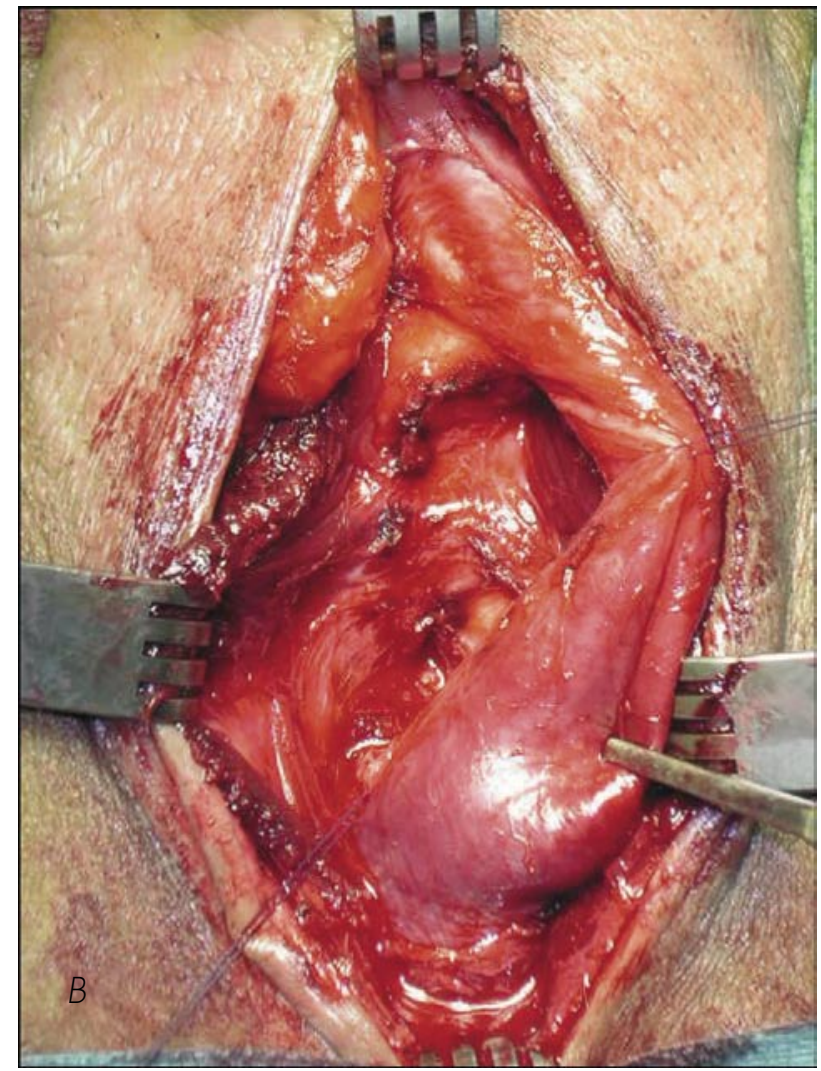

FIGURA 3A y 3B. Incisión perineal longitudinal con disección de la uretra bulbar hasta zona sana proximal y distal con rotación de la misma exponiendo su cara dorsal. 

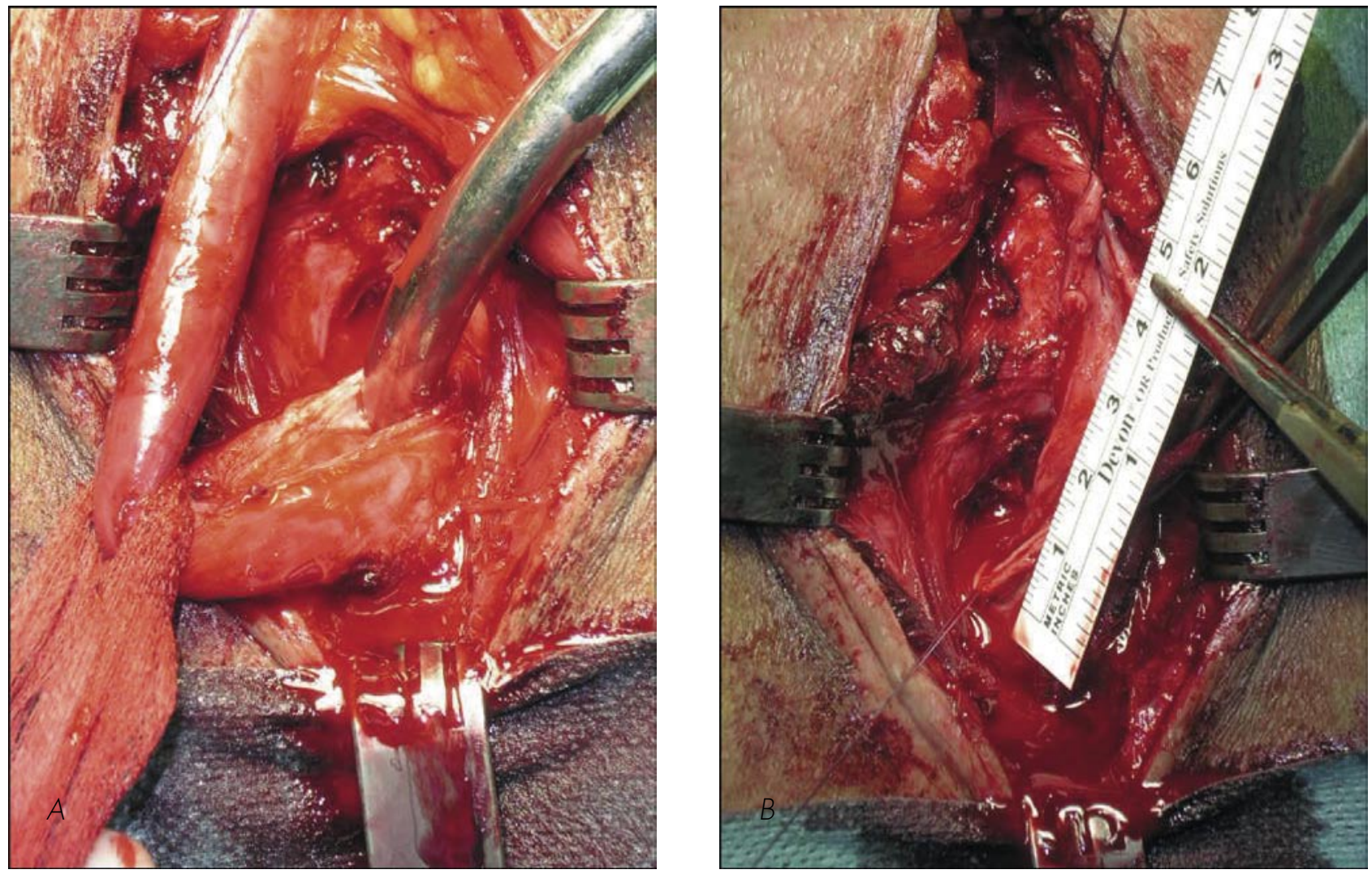

FIGURA 4. Apertura longitudinal de la estenosis hasta uretra sana y comprobación con Benique del calibre proximal y distal (A). Medida de la estenosis (B).

discontinuos y reabsorbibles. Una vez fijado se sutura a los bordes uretrales de la misma forma sobre sonda Ch 22. (Figuras 6a, 6b y 6c). Cerramos la incisión dejando un drenaje aspirativo durante 24 horas. (Figura 7).

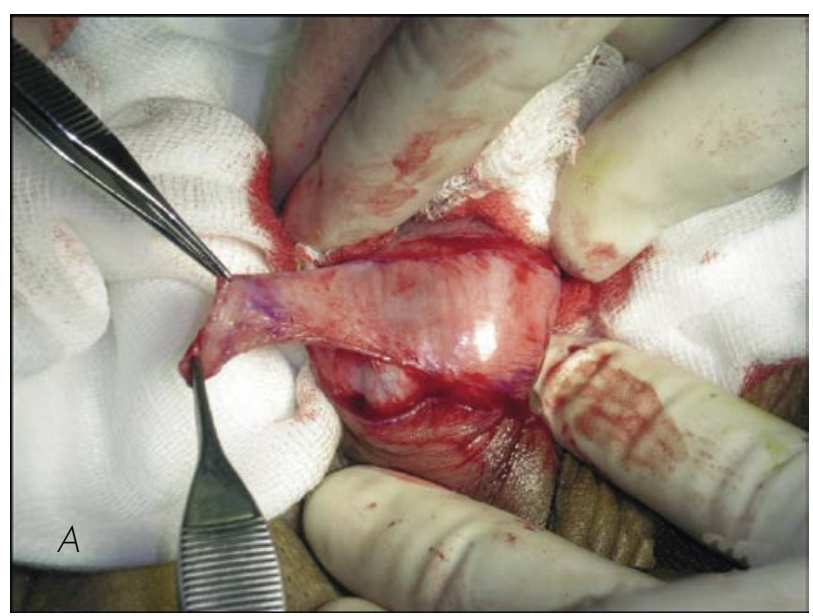

El tutor uretral se mantiene dos semanas y se retira de forma ambulatoria bajo control radiológico.

El tiempo medio de seguimiento de la serie ha sido de 22 meses (2-55). En los controles, valo-

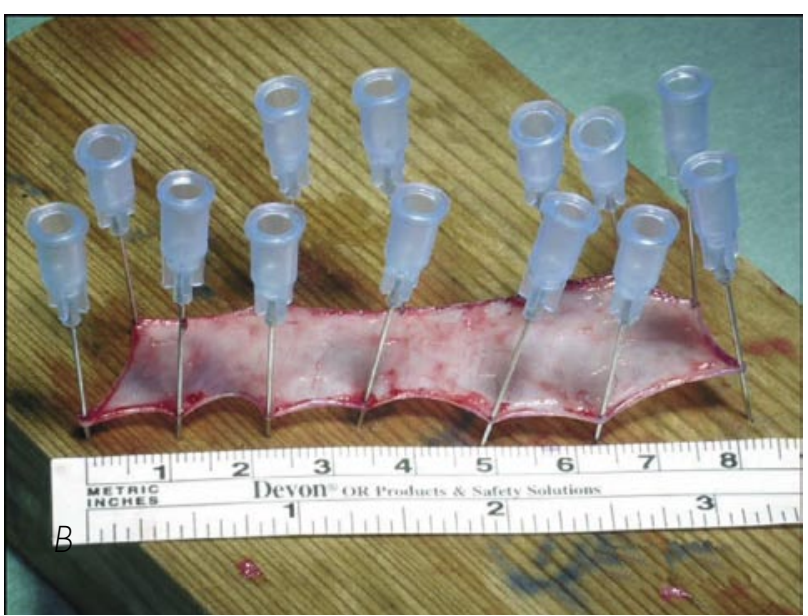

FIGURA 5. Extracción del injerto libre de mucosa prepucial (A) y su preparación en banco (B). 

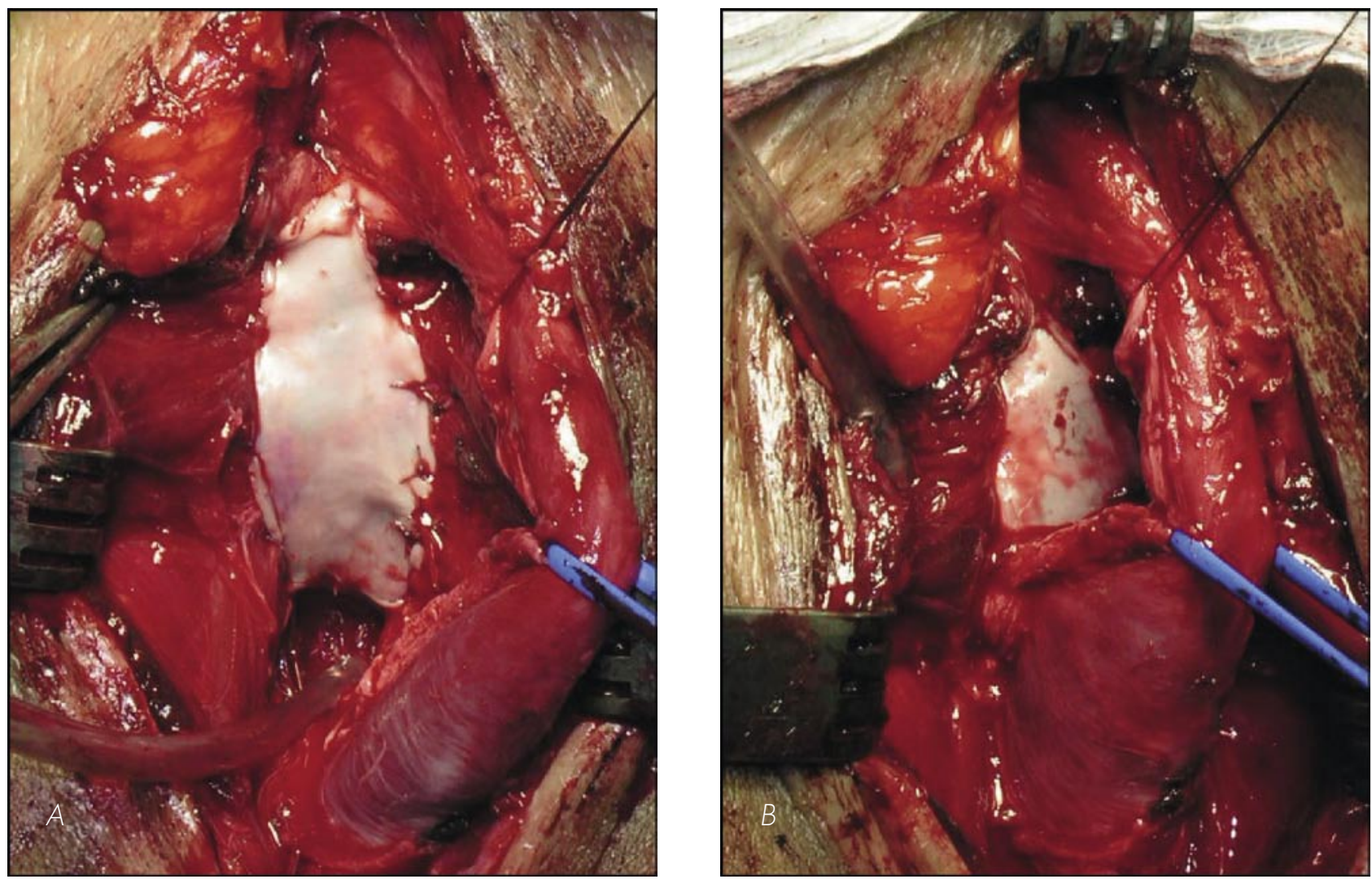

FIGURA 6. Estabilización del injerto fijándolo a albugínea de cuerpos cavernosos (A).

Anastomosis con puntos sueltos a uretra (B y C) sobre sonda Ch 20-22 French.

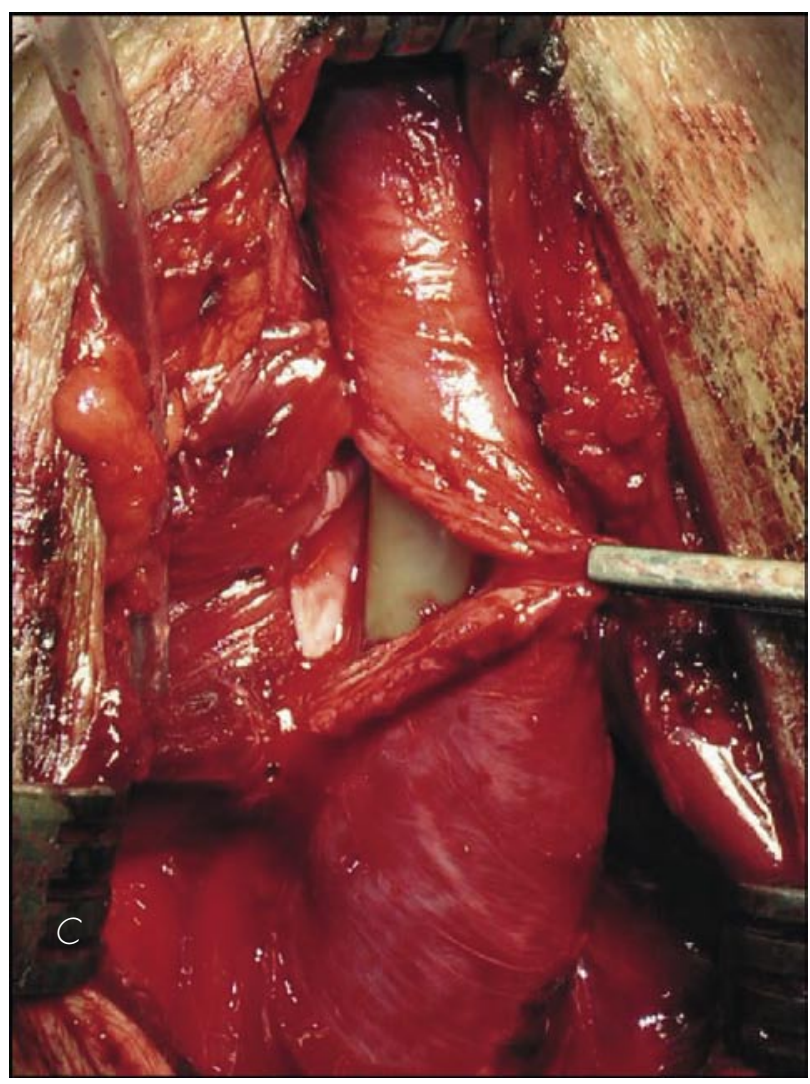

ramos la clínica y la flujometría miccional aislada. Ante la sospecha de un fracaso recurrimos de nuevo al estudio radiológico con CUMS. La primea revisión se realiza a los 2-3 meses de la cirugía, luego semestralmente durante tres años y anual pasado este tiempo.

Hemos considerado buenos resultados la existencia de un buen confort clínico con flujometrías superiores a $15 \mathrm{ml} / \mathrm{seg}$. y la no necesidad de dilataciones periódicas.

\section{RESULTADOS}

En 7 casos $(87,5 \%)$ los resultados fueron satisfactorios con una resolución definitiva de la estenosis según los criterios referidos. Sólo un paciente ha requerido un nuevo procedimiento quirúrgico (anastomosis termino- terminal) por reestenosis a nivel del extremo proximal de la uretroplastia en uretra bulbar.

No hemos encontrado complicaciones postquirúrgicas inmediatas. 


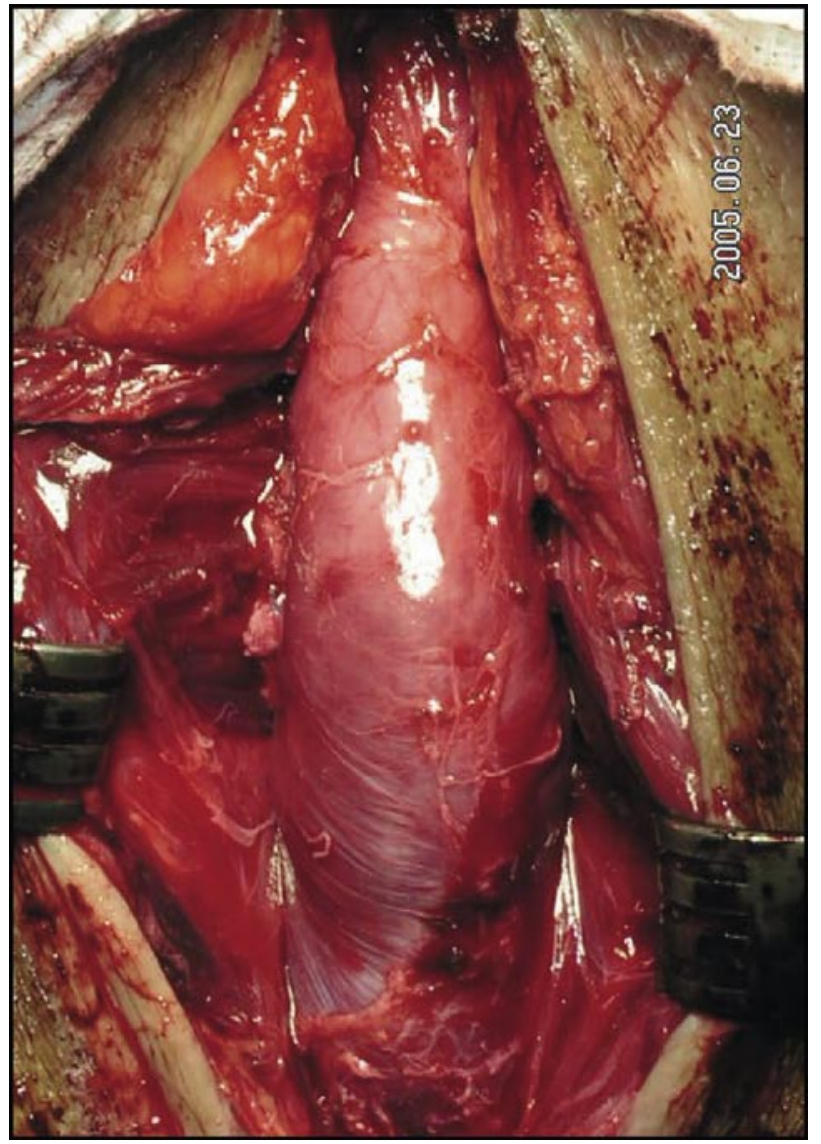

FIGURA 7. Detalle final de la Técnica.

La flujometría postquirúrgica la los 2-3 meses) ha demostrado un Flujo máximo miccional medio de $33,87 \mathrm{ml} / \mathrm{sg}$ con volumen medio de $355,28 \mathrm{ml}$. (Figura 8).

El paciente con estenosis a nivel peneano se encuentra asintomático, con Flujometría normal y sin trastornos en la erección.

\section{DISCUSIÓN}

La uretroplastia dorsal con injerto libre de mucosa fue descrita en 1995 por Guido Barbagli y colaboradores.

Los resultados obtenidos a largo plazo en series de pacientes demuestran una tasa de éxitos del 92-97\%, aunque en una serie de Barbagli caen al 92-85\% cuando el seguimiento es mayor. (Tabla I).

La elevada tasa de recurrencia de la uretrotomía interna (70\%) (8), y los buenos resultados obtenidos por la mayoría de autores, en el tratamiento de las estenosis largas y complejas a cualquier nivel de la uretra, con la uretroplastia dorsal con injerto libre de mucosa, ha motivado que, en la actulidad, se convierta en la técnica de elección considerandose ventajoso el abordaje dorsal sobre el ventral y lateral, por conseguirse un soporte fijo, facilitandose la neovascularización y evitandose el riesgo de fistulas $y$ de reestenosis. $(4,11,12)$.

Por otra parte, en el abordaje ventral, al carecer de un soporte rígido y estable, el injerto corre el riesgo de sacularse dando lugar a goteo postmiccional, infecciones y trastornos eyaculatorios $(4,9,10)$.

Sin embargo, a pesar de estas consideraciones Barbagli (14) compara los resultados obtenidos en 50 pacientes de los que en 17 sitúa el injerto en la cara ventral de la uretra, 6 en la cara lateral y 27 en la dorsal, con resultados similares $183 \%, 83 \%, 85 \%$ respectivamente).

El propio Barbagli (2) no encontró diferencias significativas entre los casos donde usó mucosa prepucial y mucosa bucal aunque un seguimiento a largo plazo permitirá sacar conclusiones al respecto,
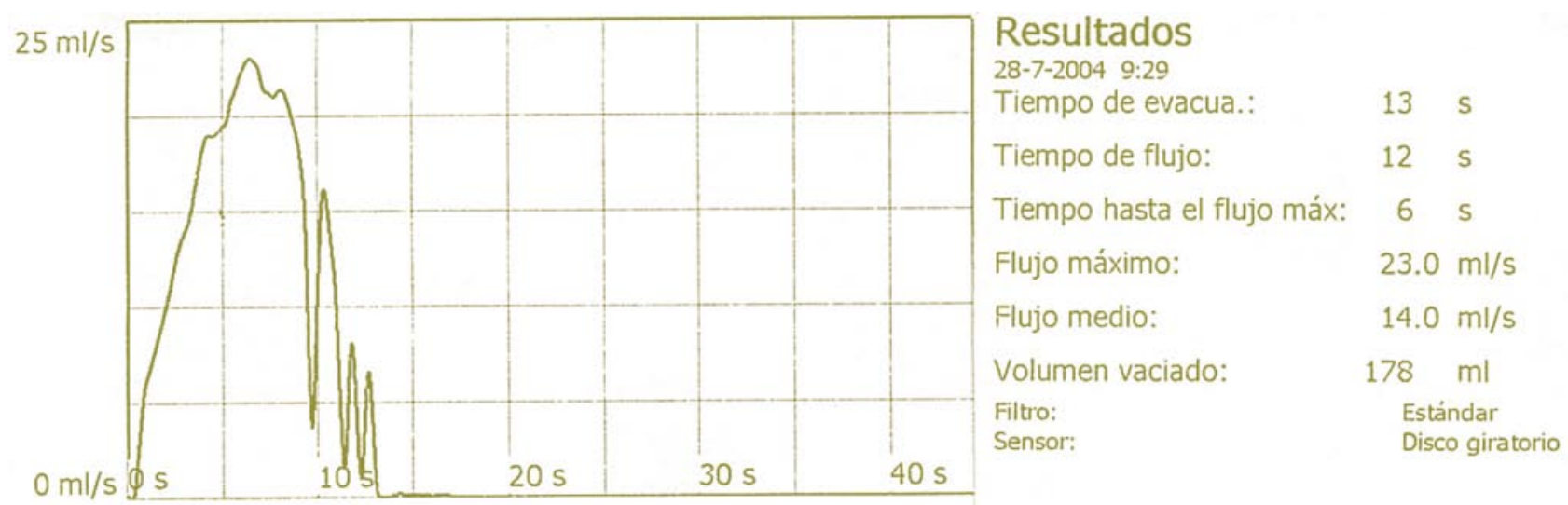

FIGURA 8. Flujometría aislada con Qmax de $23 \mathrm{ml} / \mathrm{seg}$. 


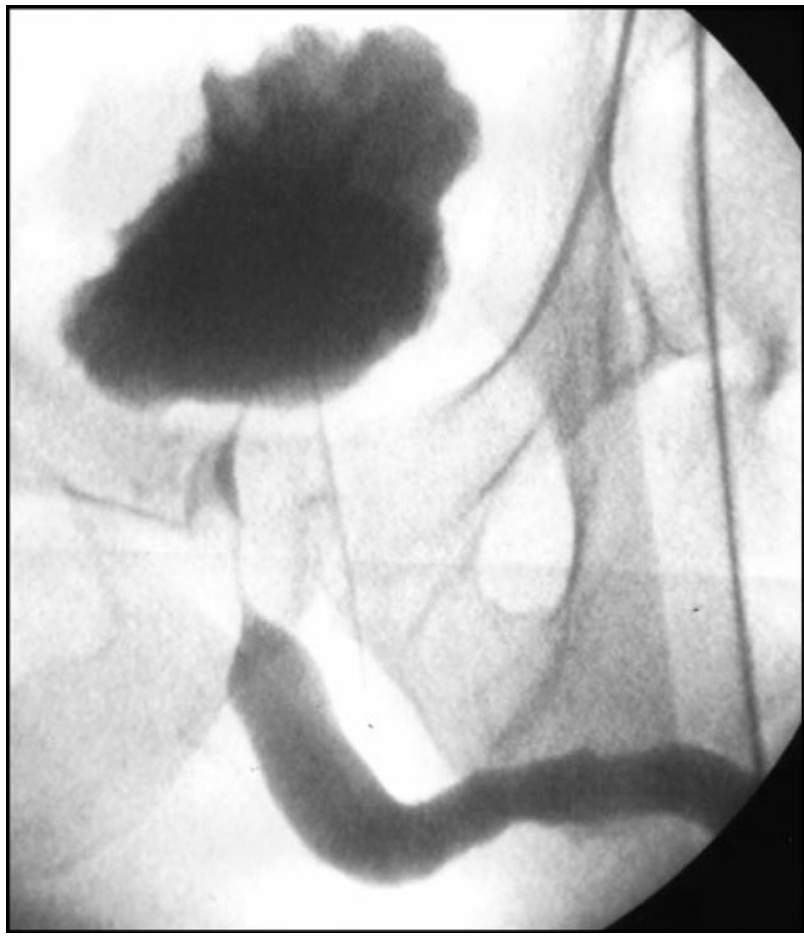

FIGURA 9. Uretrografía retrógrada una vez retirada la sonda vesical.

mientras tanto utiliza mucosa bucal o piel del prepucio según la preferencia del paciente el estado del tejido prepucial y las características de la estenosis (15). Nuestra experiencia no aporta datos al respecto pues en todos los casos hemos usado mucosa prepucial.

La indicación del procedimiento en estenosis a nivel de uretra peneana en nuestra serie (un caso) ha presentado buen resultado. Barbagli también la indica en estos casos con resultados superponibles a los que obtiene en uretra bulbar $(1,2,3)$.

El mismo autor (6) en una serie de 45 pacientes utilizando mucosa prepucial no encuentra diferencias en los resultados cuando se indicó la técnica como primera opción o secundaria a otros procedimientos. En nuestra corta experiencia tampoco existen diferencias pero su escaso número no nos permite llegar a concluir nada en este sentido.

Destacamos en el aparado de resultados la escasa morbilidad de la técnica donde no recogemos ninguna complicación postquirúrgica inmediata ni evolutiva. No existen alteraciones en la esfera sexual ni siquiera en el caso donde se trató una estenosis a nivel de la uretra peneana.

La flujometría es de gran valor en el control postquirúrgico. Llama la atención en esta serie el aumento del flujo máximo tan significativo pasando de $8,7 \mathrm{ml} / \mathrm{seg}$ a $33,8 \mathrm{ml} / \mathrm{seg}$. Una caída brusca del Qmáx es indicación para realizar estudio radiológico mediante cistouretrografía miccional seriada y valorar una posible reestenosis.

Cuando fracasa la técnica, el tratamiento dependerá de cada caso. Barbagli (6) con una tasa de fracasos del $27 \%$ (12 pacientes) recurrió a la uretrotomía interna, anastomosis termino-terminal, uretroplastia con piel y uretroplastia en dos tiempos. Seis de estos pacientes se resolvieron satisfactoriamente y los otros seis se vieron abocados a uretrostomía perineal definitiva.

TABLA I. SERIES PUBLICADAS DE URETROPLASTIA DORSAL CON INJERTO LIBRE DE MUCOSA INDICANDO EL NÚMERO DE PACIENTES, TIEMPO DE SEGUIMIENTO Y \% DE ÉXITO.

\begin{tabular}{|l|l|l|l|}
\hline Estudio & No pacientes & Seguimiento (meses) & Éxito n (\%) \\
\hline Barbagli 1996(1) & 25 & 35,8 & $23(92 \%)$ \\
\hline Barbagli 1998(2) & 37 & 21,5 & $34(92 \%)$ \\
\hline Barbagli 2001(3) & 40 & 43 & $34(85 \%$ \\
\hline Iselin 1999(4) & 28 & 19 & $27(97 \%)$ \\
\hline Guralnik 2001(5) & 29 & 28 & $27(93 \%)$ \\
\hline Barbagli 2004(6) & 45 & 71 & $33(73 \%)$ \\
\hline Parma 2005 (7) & 15 & 40 & $14(93 \%)$ \\
\hline
\end{tabular}


En nuestra serie en el caso donde fracasó la técnica, se realizó uretroplastia termino-terminal con buenos resultados evolutivos. La causa del fracaso fue una escasa apertura en la zona proximal de la estenosis inicial de ahí la importancia de llegar a uretra sana cuando se realiza la incisión dorsal.

\section{CONCLUSIONES}

La uretroplastia dorsal con injerto libre de mucosa prepucial es una técnica efectiva en estenosis de uretra larga y compleja.

Por los resultados obtenidos la consideramos técnica de primera elección en este tipo de estenosis uretrales.

Resaltamos la importancia de que el injerto se prolongue hasta ambos extremos uretrales sanos.

\section{BIBLIOGRAFÍA y LECTURAS RECOMENDADAS (*lectura de interés $y$ ** lectura fundamental)}

**1. BARBAGLI, G.; SELLI, C.; TOSTO, A. y cols.: "Dorsal free graft urethropasty". J. Urol., 156: 1447, 1996.

*2. BARBAGLI, G.; PALMINTERI, E.; RIZZO, M.: "Dorsal onlay graft urethroplasty using penile skin or buccal mucosa in adult bulbourethral strictures". J. Urol., 160: 1307, 1998.

*3. BARBAGLI, G.; PALMINTERI, E.; LAZZERI, M. y cols.: "Long term outcome of urethroplasty after failed urethrotomy versus primary repair". J. Urol., 165: 1918, 2001.

4. ISELIN, C.; WEBSTER, G.D.: "Dorsal onlay graft urethroplasty for repair of bulbar urethral stricture". J. Urol., 161 : 815, 1999.
5. GURALNIK, M.L.; WEBSTER, G.D.: "The augmented anastomotic urethroplasty : Indications and outcome in 29 patients". J. Urol., 165: 1496, 2001.

*6. BARBAGLI, G.; PALMINTERI, E.; LAZZERI, M. y cols.: "Interin outcomes of dorsal skin graft bulbar urethroplasty". J. Urol., 172: 1365, 2004.

7. PARMA, P.; DALL OGLIO, B.; SCHIAVON, L. y cols.: "Dorsal free graft uretrhoplasty according to Barbagli. Minimun follw-up of 2 year". Arch. Ital. Urol. Androl., 77: 131, 2005.

8. PENSADORO, V.; EMILOZZI, P.: "Internal urethrotomy in the management of anterior urethral strictures: long term follow-up". J. Urol., 156: 73, 1996.

9. BARBAGLI, G.; SELLI, C.; TOSTO,A.: "Preoperative surgery for recurrent strictures of the penile and bulbous urethra". J. Urol., 156: 76, 1996.

*10. WEBSTER, G.D.; ROBERTSON, C.N.: "The vascularized skin island urethroplasty: Its role and results in uretral stricture management". J. Urol., 133: 31, 1985.

11. BROWN, D.: "An operation for hypospadias". Proc. R. Soc. Med., 42: 466, 1949.

12. WEAVER, R.G.; SCHULTE, J.W.: "Experimental and clinical studies of urethral regeneration". Surg. Gynec. Obst., 115: 729, 1962.

13. BARBAGLI, G.; SELLI, C.; DICELLO, V. y cols.: "A one-stage dorsal free-graft urethroplasty for bulbar urethral strictures". Br. J. Urol., 78: 929, 1996.

*14. BARBAGLI, G.; PALMINTERI, E.; GUASÓN, G. y cols.: "Bulbar urethroplasty using bucal mucosa grafts placed on the ventral, dorsal or lateral surface of the urethra: are resuls affected by the surgical technique?". J. Urol., 174: 955, 2005.

**15. BARBAGLI, G.; PALMINTERI, E.; LAZZERI, M.: "Técnicas de uretroplastia mediante injerto dorsal de revestimiento". Anthony Atala MD and Jack W. McAninch MD, Reconstrucción uretral. Clinicas Urologicas de Norteamérica (Edición española) vol 2. McGraw-Hill Interamercana de España S.A.U. Madrid 2003. 